\title{
Going Round in Circles
}

\section{Anmerkungen zur Bewegungsrichtung in mykenischen Palastmegara}

The round hearth is a central element of Mycenaean throne rooms, not merely in spatial terms, but equally with regard to iconographic concepts and to acts - to movement in particular - in the palatial megara. On the basis of three premises -1) the non-axiality of centripetal movement in Mycenaean palaces, 2) the strongly normative form of the megaron, which suggests strong norms governing acts performed in it, and 3) the role of wallpaintings, particularly processional scenes, as what have been termed »sign-posts « - numerous elements of both the painted decoration and the architectural layout of the megara at Mycenae, Pylos and Tiryns can be understood as evidence for a primary sense of movement along a clockwise, circular route around the hearth, from the throne room's door to the throne itself. In particular, the paintings to the left and right of the Pylian throne, the hearth and the columns surrounding it are all integrated in a coherent and meaningful composition when viewed from a position opposite the throne across the hearth, possibly the closest many - even among those admitted to the megaron - ever got to the enthroned ruler.

\section{Vorbemerkung zur Alltagserfahrung und zu Prinzipien der Raumnutzung}

An American's devotion to McDonald's rests in part on uniformities associated with almost all McDonald's: setting, architecture, food, ambiance, acts, and utterances. The golden arches, for example, serve as a familiar and almost universal landmark [...] [T]he restaurants rely on their arches, dull brown brick, plate-glass sides, and mansard roofs to create a setting as familiar as home. [...] In this familiar setting, we do not have to consider the experience. We know what we will see, say, eat, and pay. ${ }^{1}$

Obschon Conrad P. Kottaks sozialanthropologische Ausführungen zur weltweit wohl bekanntesten Fast-Food-Kette insofern im Detail überholt scheinen, als der Erfolg besagter Kette längst ein globales statt ein spezifisch amerikanisches Phänomen darstellt, sind sie

Mein besonderer Dank gilt neben den Organisatoren und Teilnehmern der Konferenz »Bild - Raum Handlung« zunächst den Mitgliedern des Forschungsfeldes 4 »Orte der Herrschaft « im Cluster 3 »Politische Räume« des DAI, meinem Doktorvater Joseph Maran sowie den - akademischen wie nicht akademischen - Teilnehmern von Führungen in Tiryns für produktive Diskussionen der hier vorgestellten Ideen sowohl in abstracto als auch in situ. Ebenso bin ich für die konstruktiven kritischen Stellungnahmen als Diskutanten im Rahmen eines Aigeiros-Vortrags am DAI Athen Walter Gauß und Constance von Rüden dankbar, wobei letzterer ebenso wie Federica Gonzato zudem mein Dank für formlosere Diskussionen gilt, in denen verschiedene der hier formulierten Ideen ihren Anfang nahmen. Maria Kostoula erzeugte aus mehreren mäßigen Kopien eine publikationswürdige Abbildungsvorlage und Joseph Maran gestattete als Leiter des Tiryns-Projektes den Abdruck einer bislang unpublizierten Rekonstruktionszeichnung. Beiden danke ich ebenso für ihre graphische Hilfestellung wie allen, die mir zuliebe im Laufe der letzten beiden Jahre mit einer Kamera in der Hand in den Sanitärbereich des Flughafens Stansted vorstießen: Ann Brysbaert, Jari Pakkanen, Julietta Steinhauer und eine mir namentlich leider nicht bekannte hen-party-Teilnehmerin, auf die am Ziel ihres Fluges sicher attraktivere Motive warteten.

Kottak 1978, 77 . 


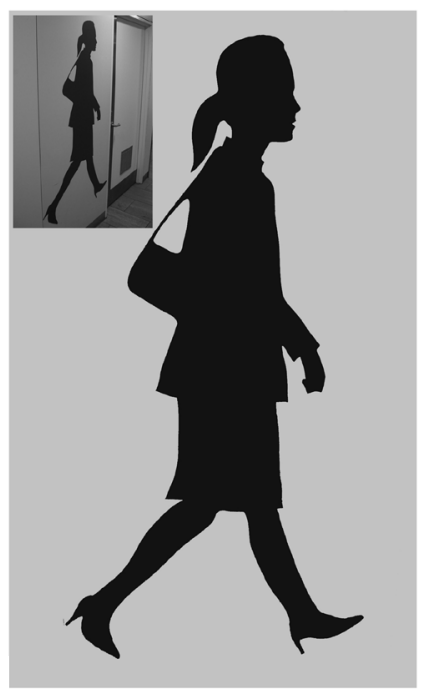

a

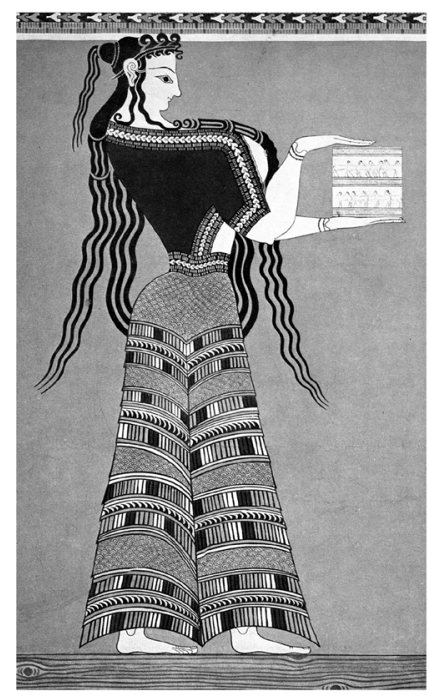

b
Abb. 1 | a: Flughafen Stansted, 21. Jh. n. Chr., Schattenrissfigur als Wegweiser zu Damentoiletten; b: Palast von Tiryns, 13. Jh. v. Chr., Wandmalerei einer Gabenträgerin aus der >großen Frauenprozession<.

im Kern ausgesprochen aufschlussreich und illustrieren in großer Klarheit einen Punkt, der für die folgenden Überlegungen zu den Palastmegara der späten Bronzezeit auf dem griechischen Festland von wesentlicher Bedeutung ist: Mit der starken Kanonisierung eines Typus öffentlich genutzter Bauten verbindet sich nicht selten eine vergleichbar starke Kanonisierung und Reglementierung - auch der Begriff »Ritualisierung « scheint keinesfalls fehl am Platze - der in diesen Bauten vollzogenen sozialen Akte.

Tatsächlich bietet auch für andere in archäologischen Interpretationen angeführte Prinzipien der Nutzung und Gestaltung von Räumen unsere Alltagserfahrung eindrückliche Illustrationen. So kann z. B. der aufmerksame Reisende im Flughafen Stansted Wandmalereien entdecken, die in ganz ähnlicher Weise eine Wegweiserfunktion erfüllen, wie dies für Prozessionsdarstellungen der ägäischen Bronzezeit in vielen Fällen angenommen wird (Abb. 1) - das Ziel, auf das die Stansteder Wandbilder hinweisen, ist zwar als Sanitärbereich ein vergleichsweise prosaisches, zweifelsohne aber eines, dem ein breites öffentliches Interesse gilt. Ich möchte die Bedeutung heutiger Alltagserfahrung für unser Verständnis der Vergangenheit indes weder überbetonen noch den Leser des Vergnügens berauben, selbst weitere illustrative Parallelen in unserer Lebenswelt zu identifizieren. Entsprechend seien zunächst, bevor ich zur Kernthese dieses Aufsatzes vorstoße - der Annahme einer im technischen Sinne rrechtsdrehenden< Bewegung um den Rundherd als kanonischer Route innerhalb der mykenischen Palastmegara -, drei Grundannahmen in neutralerer Form festgehalten, die ich anschließend als gegeben behandeln werde. 


\section{Drei Grundannahmen: Indirektheit des Zugangs, Einheitlichkeit der Megara und Malereien als Richtungsanzeiger}

1.) Obwohl, wie schon früh erkannt ${ }^{2}$, die zentripetale Bewegung hin zur Megaron-Raumfolge mit dem Thronsaal von grundlegender Bedeutung war, die sich deutlich in der architektonischen Gestaltung des Zugangsweges widerspiegelt, ist diese Route doch keinesfalls geradlinig geführt. So liegt z. B. zwar in den bekannten peloponnesischen Palästen, Mykene, Tiryns und Pylos, das zum Megaronhof führende Propylon dem Megaron gegenüber, ist jedoch stets seitlich zur Achse des Megarons versetzt ${ }^{3}$. Im Falle des Palastes von Tiryns, in dem Joseph Maran das Produkt eines in vergleichsweise kurzer Zeit umgesetzten Gesamtentwurfes sieht ${ }^{4}$, scheint sogar, wie er unter Bezug auf Johnsons Untersuchung frühneuzeitlicher englischer Burgen ${ }^{5}$ feststellt, auf Richtungswechsel an liminalen Punkten besonderer Wert gelegt zu sein ${ }^{6}$, so dass letztlich der Weg vom Burgtor zum Thronraum im Uhrzeigersinn laufend fast eine Spirale beschreibt. Auf die Verbindung von liminalen Punkten bzw. allgemeiner von Stationen entlang des Zugangswegs durch eine konsequent gestaltete Blickführung wird noch einzugehen sein.

2.) Bei allen Unterschieden in der konkreten Plangestaltung liegen den bekannten mykenischen Palastanlagen, worunter angesichts unseres unvermeidlich lückenhaften Kenntnisstandes zu Theben zunächst wiederum die genannten drei peloponnesischen Paläste verstanden sein sollen, offenbar gemeinsame Gestaltungsideen zugrunde. Das wohl wirkungsstärkste dieser Konzepte findet seinen Ausdruck in der Megaron-Raumfolge (Abb. 2). Diese war zwar unterschiedlich durch Seiteneingänge mit dem Rest der Palaststruktur verbunden - in Mykene durch eine Tür in der offenen Vorhalle, in Tiryns durch eine Tür im inneren Vorraum und in Pylos wohl durch zwei Türen ebenfalls im inneren Vorraum -, weist jedoch an allen drei Orten dieselbe Gliederung in Vorhalle, Vorraum und Thronsaal auf, hinzu kommen nur geringfügig variierende Abmessungen und vor allem eine offenbar kanonische Ausstattung des Thronsaales mit einem Rundherd zwischen vier Säulen in der Mitte und einem Thronplatz auf der vom Eingang aus rechten Seite. Der Thronplatz ist natürlich in Mykene durch das Abrutschen des entsprechenden Teils der Anlage nicht erhalten, dass seine Rekonstruktion unbestritten ist, mag aber die Eingängigkeit des Konzeptes - zumindest für Archäologen - belegen. Die vielleicht

2 z. B. Rodenwaldt (1919, 92), der mit spürbarer Begeisterung notiert, »[w]ie [...] in Tiryns der Eintretende mit genialer Ausnutzung des ansteigenden Terrains von einem Vorhof zum anderen, von einem Propylon zum anderen geführt wird, bis sich zuletzt die mächtige Fassade des Megarons in eindrucksvoller Symmetrie mit den flankierenden Säulenhallen in der Achse des Hofes erhebt, wie alles auf diese Schlußwirkung berechnet ist, wie einer Richtung, einem Ziel alles andere sich unterordnet«.

3 Maran 2006, 82 einschl. Anm. 62.

4 Maran 2004, 283; vgl. Küpper 1996, 111.

5 Johnson 2002, 73-85, bes. 83 .

6 Maran 2006, 83; vgl. Anm. 21. 

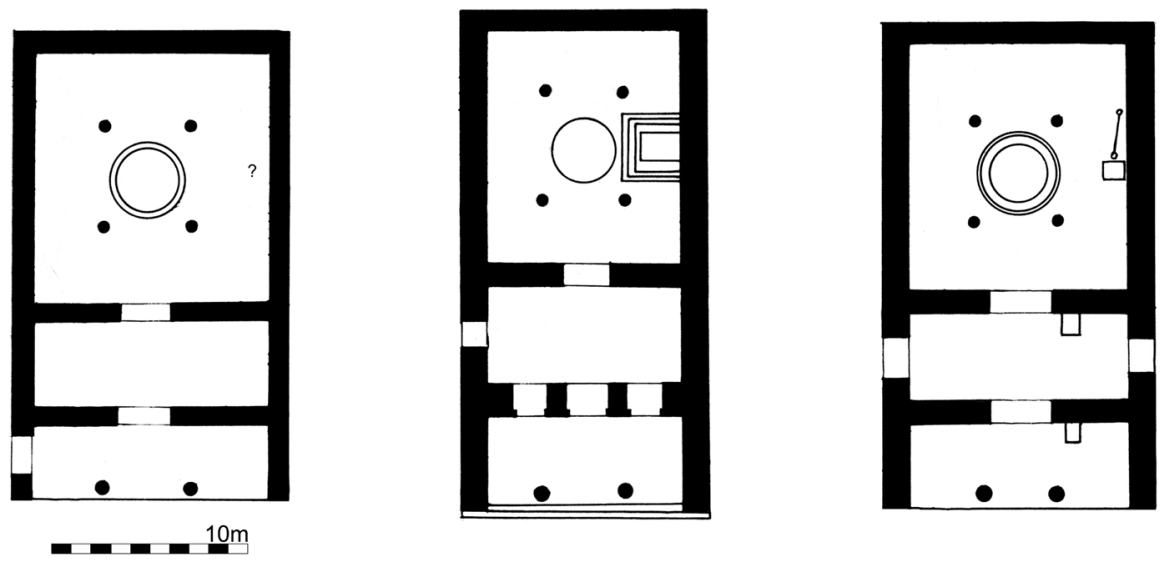

Abb. 2 | Grundrisse der Palastmegara (von links nach rechts) von Mykene, Tiryns (großes Megaron) und Pylos (M. $1: 500)$.

aufschlussreichsten Vergleichsfälle bieten derzeit der im letzten Jahrzehnt ausgegrabene Herren- oder Herrschersitz von Dimini und das kleine Tirynther Palastmegaron, die über eine Vereinfachung des bestehenden Konzeptes andeuten, welche Elemente am ehesten als verzichtbar galten? ${ }^{7}$.

Diese Einheitlichkeit in der Gestaltung von Megaron-Raumfolge und Thronraum ist im Folgenden in zweierlei Hinsicht von Bedeutung: Zum einen gibt sie Anlass zu der Vermutung, dass mit der streng kanonisierten Form auch streng kanonisierte Nutzungsformen verbunden waren, was z. B. eine Hauptbewegungsrichtung innerhalb des Thronraumes plausibel erscheinen lässt. Zum anderen und in Zusammenhang damit macht sie die Übertragung von Beobachtungen, die an einem der Palastorte aufgrund der spezifischen Befundlage möglich sind, auf die anderen Palastorte wahrscheinlicher als in vielen anderen Fällen: Wenn Analogieschlüsse grundsätzlich die Gefahr mit sich bringen, durch Verallgemeinerungen das jeweils Besondere und Andersartige aus dem Blick zu verlieren, bleiben sie dort ungefährlich, wo keine oder nur geringe Normabweichungen zu erwarten sind. Dies ist $u$. a. auch in Hinsicht auf die dritte hier der Diskussion zugrunde gelegte Annahme von Bedeutung.

3.) Auf das Megaron bzw. den Thronsaal hin orientierte Menschen- und insbesondere Prozessionsdarstellungen können für die zeitgenössischen Benutzer der Palastarchitektur, d. h. in diesem Falle besonders für die Besucher des Palastes, als Wegweiser auf

7 Damit würden sich beide auch als Ausgangspunkt für eine Diskussion anbieten, die versucht, die zu Recht oft beklagte Unschärfe im Gebrauch des Megaronbegriffes nicht durch dessen Aufgabe zu beseitigen (Darcque 1990; Jung 2000), sondern durch eine sorgfältige Definition, die sich an einem frühgeschichtlichen Verständnis des Bautypus orientiert; freilich verdeutlicht schon der Umstand, dass in Tiryns die Räume XXI und XXII in mancher Hinsicht wiederum als verkürzte Replik des kleinen Megarons erscheinen, die Schwierigkeiten einer solchen Diskussion. 


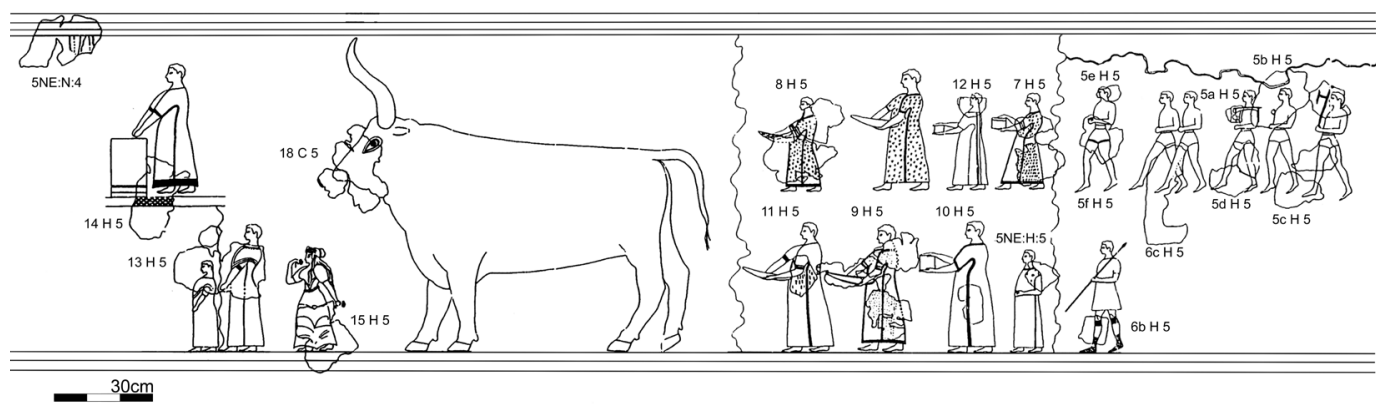

Abb. 3 | Rekonstruktion der Prozessionsszene im Vorraum 5 des Palastmegarons von Pylos nach Lucinda McCallum (M. $1: 30)$.

dem Weg zum Thron bzw. zum inthronisierten Herrscher gedient haben oder können zumindest allgemeiner als Indiz für einen regelmäßigen Fluss des Besucherverkehrs in die entsprechende Richtung verstanden werden. Das klassische Beispiel für diese These bietet die ehemals im inneren Vorraum 5 des pylischen Megarons angebrachte, Wandmalereifragmente und Fragmentkomplexe $5 \mathrm{H} 5$ bis $15 \mathrm{H} 5$ sowie $18 \mathrm{C} 5$ umfassende Prozessionsdarstellung (Abb. 3$)^{8}$. In Anlehnung an Mark Camerons Deutung knossischer Wandmalereien ${ }^{9}$ hat insbesondere Lucinda McCallum in ihrer eingehenden Studie der Megaron-Fresken eine Deutung als »sign-post« vertreten und in direkter Entsprechung auch für eine menschliche Darstellung in der äußeren Halle 1 des Propylons vorgeschlagen $^{10}$, also an einer weiteren wichtigen Station auf dem Weg zum Thron, einem liminalen Punkt im Sinne Marans. Zur Begrifflichkeit ist hierbei anzumerken, dass der Terminus »sign-post« insofern nicht problemlos ist, als er eine unzweifelhaft normative Funktion impliziert und dadurch die Möglichkeit ausblendet, dass die Fresken tatsächliche Prozessionen und deren Bewegungsrichtung unter Umständen schlicht kommemorierten; eine Funktion als Zeichengeber ist durchaus wahrscheinlich, aber nicht Voraussetzung für eine archäologische Analyse der Bewegungsrichtung. Überzeugend ist hingegen McCallums weiterführende Annahme einer Verbindung des Prozessionsfreskos in Vorhalle 5 mit der im Thronraum 6 rekonstruierten Bankettszene mit Leierspieler als Elemente einer narrativen Folge, wobei sie konkret an eine Handlungsabfolge im Rahmen eines religiösen Festes denkt $t^{11}$.

8 Lang 1969, 64-68. 109. 192 Taf. 119; McCallum 1987, 195-197 Taf. 8 a-c.

9 Cameron 1970, 165.

10 McCallum 1987, 70-71. 119.121-122.

$11 \quad$ McCallum 1987, 108. Im Detail ist McCallums Auffassung dahingehend zu korrigieren, dass in den Malereien im Thronraum nach neueren Erkenntnissen kein Bulle zu identifizieren ist (Stocker - Davis 2004, 190), womit der von McCallum vermeintlich identifizierte Bulle als inhaltlich mit der unstrittigen Bullendarstellung im Vorraum verbundenes Element zwangsläufig ausfällt; insgesamt bleibt ihre Deutung jedoch schlüssig. 
Dass einer ähnlichen Funktion als normativer und/oder kommemorativer Richtungsanzeiger im Falle der nicht in situ am ursprünglichen Anbringungsort aufgefundenen großen Tirynther Frauenprozession einige Wahrscheinlichkeit zuzubilligen ist, hat zuletzt Maran überzeugend dargelegt $\mathrm{t}^{12}$. Weniger plausibel erscheint eine entsprechende Deutung bei der ebenfalls großformatigen Frauenprozession aus dem sog. Haus des Kadmos in Theben ${ }^{13}$ : Zwar bleibt durch die bestehende Unklarheit hinsichtlich des Gesamtplans der Anlage und der Position eines mutmaßlich vorhandenen Megarons jedwede Aussage notwendigerweise eine vorläufige, aber der Umstand, dass Raum N als Fundort der Freskenreste auf zwei Seiten von Korridoren und auf der dritten wohl von einer Außenmauer begrenzt wurde, lässt diesen Raum kaum als Durchgangsraum auf dem Weg zur zentralen Raumfolge des thebanischen Palastes erscheinen.

\section{Der Fragmentkomplex 45 H 6 als Denkanstoß}

Von diesen drei Annahmen - Indirektheit des Zugangsweges zum Thron, Einheitlichkeit von Megaronplan und -nutzung sowie Richtungsweiserfunktion der Darstellung von Prozessionen zumindest in Pylos - dürfen die beiden letztgenannten zumindest im Kern als weithin akzeptierte Forschungsmeinung gelten, während die hier zuerst diskutierte, in die Forschungsdiskussion aber zuletzt in der umrissenen Form eingeführte Auffassung sich meines Erachtens rasch als solche etablieren dürfte. So ist es bemerkenswert, dass trotz ihrer unstrittigen Natur diese drei Punkte bereits eine hinlängliche Ausgangsbasis bieten, um in der Betrachtung einer aufgrund ihrer schlechten Erhaltung bislang wenig beachteten Wandmalereiszene, dem Fragmentkomplex 45 H 6 aus dem Thronsaal von Pylos $^{14}$, zu einer neuen und unerwarteten Perspektive auf die Frage nach Handlungsformen und Bewegung in den mykenischen Thronsälen zu gelangen, die anschließend durch weitere kontextuelle Beobachtungen zu bestätigen sein wird.

Neben Resten weißer Gewänder mit farbigen Diagonalstreifen überliefert der in seinen größten Maßen nur 13,5 cm hohe und 23,5 cm breite, stark beschädigte Fragmentkomplex zwei rot angegebene Unterarme, nach Mabel Lang die dem Betrachter zugewandten und demnach rechten Unterarme zweier nach rechts schreitender Männer ${ }^{15}$. Das wenige,

\footnotetext{
12 Maran im Druck.

13 Reusch 1956, bes. 3-5 einschl. Abb. 1.

14 Auf eine Abbildung von 45 H 6 verzichte ich mit Bedauern, letztlich aber aus zwingendem Grund: Die bei Lang (1969, Taf. 29) reproduzierte Darstellung ist aufgrund des sehr schlechten Zustandes des Stücks kaum informativ. Eine zusätzliche Reinigungs- und Restaurierungsarbeiten umfassende Neubearbeitung der Megaronfresken ist derzeit im Gange und hat im Laufe dreier Aufarbeitungskampagnen bereits gute Fortschritte erbracht, wird aber voraussichtlich erst nach vergleichbaren Arbeiten zu den Malereien aus Raum 64 publiziert werden (pers. Mitt. Sharon Stocker, 02.07.2010; vgl. Anm. 22); neue Photographien der Malereien aus Thronraum 6 und somit auch von 45 H 6 sind deshalb derzeit noch nicht verfügbar.
}

$15 \quad$ Lang $1969,81.195$. 
was von den Gewändern erkennbar ist, scheint am ehesten einen Vergleich mit den kurzärmeligen, aber knöchellangen Gewändern zu erlauben, die in der Prozessionsdarstellung in Vorhalle 5 offenbar ranghöhere Teilnehmer kennzeichnen ${ }^{16}$. Zudem spricht für eine Verbindung mit dieser Szene die zu rekonstruierende Größe der Figuren in 45 H 6: Lang gibt diese als »about the same height as the larger figures in the Vestibule Procession « $\mathrm{an}^{17}$, McCallum sieht hiervon leicht abweichend, vielleicht auch nur in der Formulierung, die Figuren in $45 \mathrm{H} 6$ als »slightly shorter than the largest robed figures from the Vestibule 5 procession ${ }^{18}$; angesichts der zwischen 27 und $40 \mathrm{~cm}$ variierenden Höhe der Figuren in der Prozessionsszene in Raum 5 darf damit eine Höhe innerhalb der dort vertretenen Schwankungsbreite als sicher und eine eher an deren oberen Ende liegende als sehr wahrscheinlich gelten. Eine Überlappung zweier Figuren, wie sie 45 H 6 nahezulegen scheint, ist zwar untypisch für die Vorraumszene, tritt aber zumindest einmal im Fragment $6 \mathrm{c} \mathrm{H} 5$ auf ${ }^{19}$. Insgesamt erscheint es somit plausibel, in $45 \mathrm{H} 6$ eine in nur geringen Resten erhaltene Darstellung zu sehen, die inhaltlich mit der bekannten Prozessionsszene aus Vorraum 5 eng zusammenhängt und diese, sofern der Auffindungs- dem ehemaligen Anbringungsort von 45 H 6 entspricht, innerhalb des Thronsaales 6 aufgreift oder fortsetzt.

Folglich ist der Anbringungsort der durch 45 H 6 vertretenen Darstellung von direktem Interesse für die Frage nach der Bewegungsrichtung innerhalb des Megarons. In Hinsicht auf letztere ging McCallum plausibel davon aus, dass »the location and orientation of the festival scenes [d. h. Prozessions- und Bankettdarstellung in Raum 5 bzw. 6] were consistent with circulation patterns in the megaron «, wobei sie ebenso schlüssig als Beispiel anfügte, dass »the Vestibule procession moved left, in the direction of the entrance to Room 6 «. Die hieran wiederum anschließende Vermutung, dass »once inside Room 6, one would probably have turned right, toward the (NE) throne wall on which the sacrifice and banquet scenes were painted $\aleph^{2 \circ}$, steht hingegen in direktem Widerspruch zu dem, was der von Lang und McCallum übereinstimmend vorausgesetzte Anbringungsort von 45 H 6 aus Sicht des Eintretenden links der Tür an der Südostwand des Thronsaales nahelegt ${ }^{21}$ : Ausgehend von McCallums eigener Prämisse und entgegen ihrer Schlussfolgerung - und vielleicht unserer Intuition - lassen der genannte Anbringungsort von $45 \mathrm{H} 6$ und die Orientierung der dargestellten Figuren nach rechts und somit von der Thronraumtür und damit auch der Thronwand weg nur den Schluss zu, dass sich Besucher beim Eintritt in den Thronsaal nach links und somit nicht direkt zum Thron wandten.

16 McCallum 1987, 113-117.

17 Lang $1969,81$.

18 McCallum 1987, 103.

19 Lang $1969,65$.

20 McCallum 1987, 123-124 Zur vermeintlichen »sacrifice scene« vgl. Anm. 11.

21 Lang 1969, 195; McCallum 1987, 103. 
Wenn wir diese Bewegung in Gedanken fortsetzen, ergibt sich natürlich eine Kreisbewegung im Uhrzeigersinn um den Herd herum, womit die zentrale und im Folgenden näher auszuführende These dieses Aufsatzes erreicht ist. Zunächst sind allerdings noch zwei qualifizierende Anmerkungen zu 45 H 6 vonnöten: Zum einen ist zu bedenken, dass der Fragmentkomplex nicht an der Wand, sondern verstürzt vor dieser gefunden wurde. Verschiedentlich wird in der Publikation der pylischen Fresken bei anderen Funden die Möglichkeit eines Versturzes vom Nachbarraum her diskutiert - und dass eine Szene mit Figuren, die denen der nach links gewandten Prozessionsszene, die sich in Vorraum 5 rechts der Tür zum Thronsaal fand, in ihrer Größe entsprechen und nach rechts bewegt dargestellt sind, in diesem Sinne im südwestlichen, also links der Tür zu Raum 6 gelegenen Abschnitt der Wand von Raum 5 einen sinnvollen Platz fände, steht außer Frage. Weder findet sich aber dieser Verdacht, dass also die Figuren von 45 H 6 gerade auf der Rückseite der Wand angebracht waren, auf der sie hier vorausgesetzt werden, in irgendeiner Weise bei Lang oder auch McCallum angedeutet, noch bietet eine der beiden aus ihrem eingehenden Studium des Materials heraus auch nur ein indirektes Indiz hierfür ${ }^{22}$. Zum anderen ist einschränkend zu bedenken, dass im Gegensatz zum Prozessionsfresko im Vorraum 5 und einer eventuell vergleichbaren Darstellung in der äußeren Halle des Propylons 1 die durch 45 H 6 repräsentierte Szene beim Betreten des Thronraumes nicht direkt im Blickfeld des Besuchers lag, sondern von diesem höchstens peripher wahrgenommen werden konnte. Zwar bereitet dies bei einer Ansprache als »sign-post« Schwierigkeiten, wie bereits erläutert scheint diese Bezeichnung aber in sich bereits problematisch, da sie eine mögliche kommemorative Funktion der Wandmalereien, die für eine Interpretation der Bewegungsrichtung ebenso von Interesse ist wie die implizierte normative Funktion und mit der angenommenen relativen Positionierung von $45 \mathrm{H} 6$ und Besuchern voll und ganz vereinbar ist, von vornherein ausblendet.

Beweiskraft ist demnach dem Fragmentkomplex 45 H 6 in der ohnehin einem tatsächlichen Beweis kaum zugänglichen Frage der Bewegungsrichtung im Thronsaal nicht zuzusprechen. Er soll hier aber auch nicht mehr bieten als ein Indiz und zugleich den Anstoss geben zu weiteren Überlegungen, die hoffentlich in Verbindung mit diesem ersten Indiz ebenso wie aus sich selbst die vorgestellte These einer Bewegung im Uhrzeigersinn zumindest plausibel machen. Diese Überlegungen betreffen das Layout und die weitere Dekoration der Megara sowie unsere Vorstellungen von der mykenischen Herrscherideologie.

22 In einem online zugänglichen Vorbericht zu neuen Arbeiten im Magazin des Museums in Chora (vgl. Anm. 14) deutet Brecoulaki (2005) die Möglichkeit einer Verlagerung von 45 H 6 aus dem Vorraum 5 an; da sie hierfür aber keine Gründe angibt, die über Ähnlichkeiten zwischen 45 H 6 und den Malereien in Vorraum 5 hinausgehen, besteht derzeit, d. h. bis eine schlüssige Begründung für die Annahme einer Verlagerung publiziert wird, kein Anlass, am bisherigen Publikationsstand zu zweifeln. Dennoch ist es angemessen, gerade in diesem Kontext zu betonen, dass 45 H 6 zwar den Anstoß für die hier vorgestellten Überlegungen darstellt, nicht jedoch den Schlüsselbeleg der vorgestellten These, die sich auf eine Mehrzahl kontextueller Betrachtungen stützt (s. u.). 


\section{Die Evidenz der baulichen Gestalt der Palastmegara}

Hinsichtlich des Layouts ist eine einfache Schreibtischübung geeignet, zunächst die Gegenannahme einer direkten Bewegung von der Thronsaaltür zum Thronplatz kritisch zu beleuchten. Verbindet man auf einem Plan des Tirynther Megarons die Mittelpunkte der Türschwelle des Thronraumes 7 und des durch ein Podest markierten Thronplatzes durch eine gerade Linie, schneidet diese die südöstliche Säule, d. h. die Sichtlinie wird von dieser Säule unterbrochen. Zwar darf man in Analogie zu Pylos, wo die entsprechende Sichtlinie zwischen Schwelle und dem hier weniger weit in den Raum hineinragenden Thronplatz nicht gestört wird, wohl davon ausgehen, dass der Thron auf dem Podest kaum mittig, sondern von der Mitte aus zur Wand hin versetzt stand. Dennoch verbleibt die entsprechende Säule in beiden Fällen als Sichthindernis nahe der Mitte des Blickfeldes, wodurch unter Umständen eine volle Sichtbarkeit des Besuchers für den Thronenden, als den ich in der Folge der Einfachheit halber den wanax voraussetzen werde ${ }^{23}$, erst gegeben wäre, wenn beide sich schon fast in unmittelbarer Nähe zueinander befinden. Um zudem dem Herrscher en face gegenüberzutreten, verbliebe dem Besucher zwischen Herd und Thronpodest nur ein geringer Raum, in Pylos wenig mehr als zwei, in Tiryns kaum mehr als ein Meter; eine solch fast schon intime Nähe zum wanax dürfte selbst unter denjenigen, die überhaupt ins Megaron vorgelassen wurden, nur einem kleinen Kreis gestattet gewesen $\operatorname{sein}^{24}$. Dagegen bietet der nach einer Viertelumkreisung des Herdes im Uhrzeigersinn erreichte Punkt gegenüber dem Thron einerseits eine ungestörte Sichtbeziehung zum Thron, für die die Säulen keine Hindernisse, sondern eine Rahmung darstellen, und andererseits, durch die Nutzung der Breite des Megarons, eine eher angemessene Distanz für ein Respekt bezeugendes Grüßen des Herrschers und das förmliche Eintreten in die Interaktion mit diesem ${ }^{25}$.

Der Umstand, dass der Besucher schon vor dem Erreichen dieses Punktes durch die Säulen hindurch und vielleicht aus den Augenwinkeln einen Blick auf den wanax erhaschen

23 Die vorgestellten Überlegungen werden durch diese Annahme nicht berührt und bleiben in gleicher Weise gültig, wenn z. B. die Königin oder eine Inkarnation der potinija auf dem Thron vermutet wird (Rehak 1995, bes. 97. 117).

24 Vgl. Halls $(1966,107-122$ Taf. 3-12) Unterscheidung von »intimate«, »personal«, »social« und »public distance $\ll$. Nach Halls Terminologie wäre die Distanz zwischen dem Herrscher und dem Besucher jenseits des Herdes als »public distance - close phase« einzuordnen, während ein zwischen Herd und Thron stehender Besucher sich dem Herrscher bis in den Nahbereich der »social distance « oder sogar schon in den Fernbereich der »personal distance« genähert hätte. Zu beachten bleibt indes Halls $(1966,121)$ Betonung des jeweils kulturell spezifischen Umgangs mit solchen Zonen, wodurch nach westlichem Verständnis >öffentliche< Kontakte in anderen Kulturen durchaus als >persönlich< verstanden und mithin in >persönlicher< Distanz verhandelt werden können; dieser Vorbehalt wird aber seinerseits durch die Erkenntnis relativiert, dass in der mykenischen Palastarchitektur vielfach und sehr bewusst Mittel der Distanzierung eingesetzt wurden.

25 Eine erste Respektsbezeugung bereits auf der Schwelle, d. h. beim Eintritt in den Thronsaal, erscheint durchaus denkbar, ein dort entbrachter Gruß als Beginn eines wie auch immer gearteten Austausches hingegen kaum. 


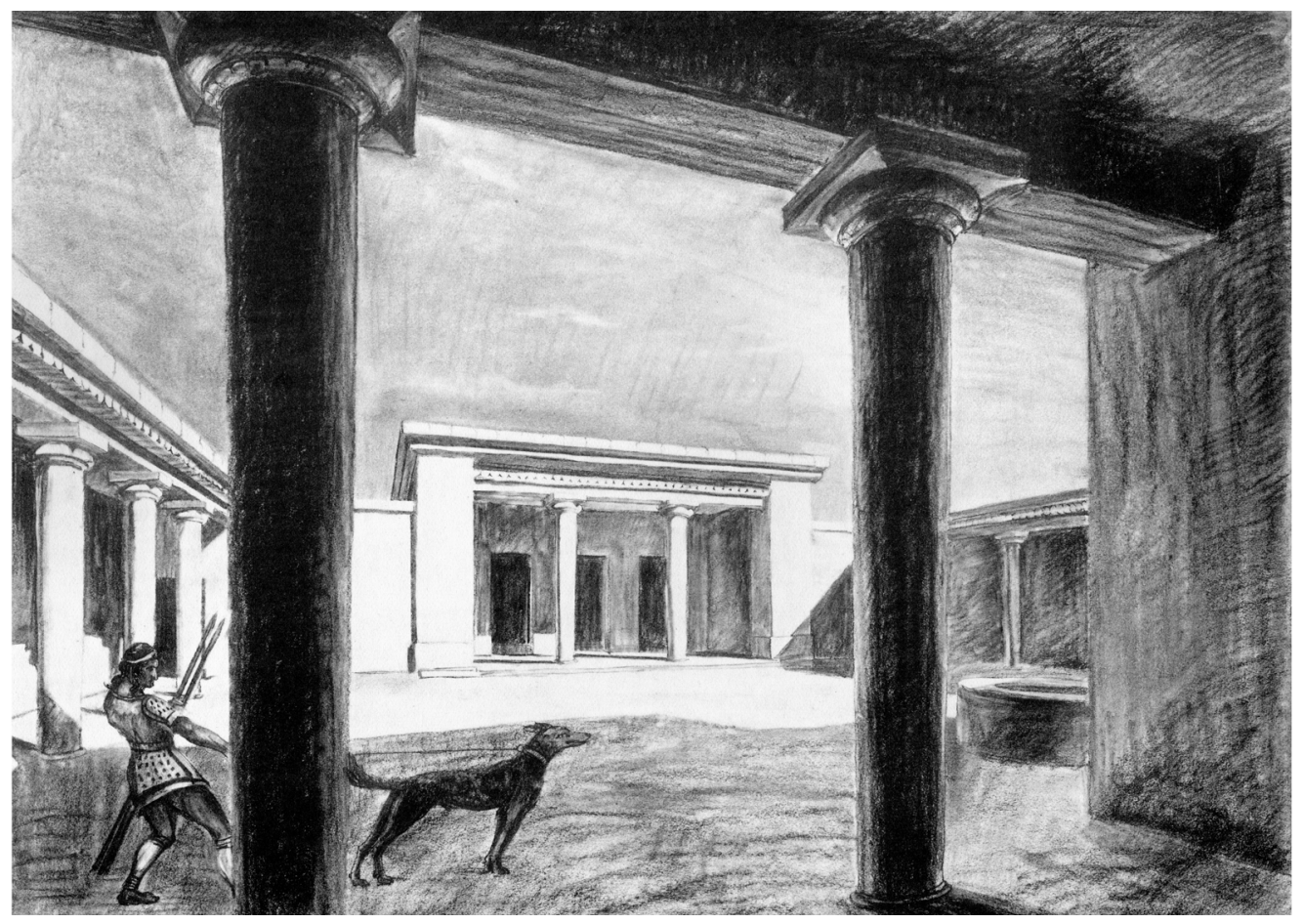

Abb. 4 | Rekonstruktion des Blicks aus dem kleinen Propylon auf das große Megaron im Palast von Tiryns, frühere Fassung der bei Müller (1930, Taf. 42) publizierten Ansicht.

konnte, mag hierbei durchaus als Fortsetzung der Blickführung auf dem Weg zum Megaron gelten, die zumindest in Tiryns sehr sorgfältig gestaltet war und dem Besucher regelmäßig einen Blick auf die nächste Station auf dem Weg zum Thronsaal, aber nicht oder nur selten darüber hinaus erlaubte ${ }^{26}$ : von außen auf Rampe und Burgtor, vom Burgtor auf das Haupttor - und nur, wenn dies offen war, auf das Tor beim äußeren Vorhof -, vom Haupttor zu letztgenanntem Tor ${ }^{27}$, von dort auf das große Propylon, das seinerseits wieder subtil in Richtung des kleinen Propylons verkippt war, vom kleinen Propylon auf das Megaron (Abb. 4), dessen Fassadengestaltung die Propyla bereits angedeutet hatten.

26 Vgl. Maran 2006, 83. Hier ergibt sich auch der eine Gegensatz, der in Ergänzung zu Marans zuvor erwähntem erhellenden Vergleich mit der Darstellung Johnsons zu vermerken ist: Während in den von Johnson diskutierten englischen Anlagen die indirekte Wegeführung auch dem Zweck dient, viele Ansichten des Baues zu präsentieren, also auf Sichtbarkeit ausgelegt ist, scheint die indirekte Wegeführung in Tiryns mit der Blickführung auf die nächste Station, aber eben nicht darüber hinaus, eher auf ein Verbergen und allmähliches Enthüllen ausgerichtet zu sein.

27 Zur Frage, ob das Tor am äußeren Vorhof im letzten Bauzustand des Palastes noch Bestand hatte, vgl. Müller 1930, 26. 193. Wenngleich ein Torverschluss nicht belegbar ist, scheint eine gliedernde Funktion eines fortbestehenden Torbaues plausibel zu sein. 
Dies lässt sich fortsetzen mit dem Blick in den Thronsaal, aber noch nicht auf den Thronenden, aus Vorhalle und Vorraum des Megarons und dann eben vielleicht einem ersten flüchtigen Blick auf den Herrscher, bevor der Besucher in eine volle visuelle Kopräsenz mit dem wanax eintrat, der wiederum für bestimmte Besucher eine engere räumliche Kopräsenz gefolgt sein mag.

\section{Die Evidenz der Ausschmückung der Palastmegara}

Was sich solchermaßen in der festen Grundgestalt von Mauern und Stützen als diachron stabilen fixen Elemente andeutet, findet weitere Bestätigung, über die Evidenz des Fragmentkomplexes 45 H 6 hinaus, in den Wand- und Bodenmalereien von Pylos und Tiryns, die als diachron nicht stabile fixe Elemente eine detailliertere Definition des architektonischen Handlungsrahmens erlauben ${ }^{28}$. Nur am Rande sei hierbei erwähnt, dass im Thronsaal von Pylos einige wenige Felder innerhalb des Quadratrasters der Fußbodenbemalung neben ihrer malerischen Ausschmückung auch Ritzungen aufweisen, in denen bereits die Ausgräber mögliche Standortmarkierungen für Teilnehmer am Hofritual sahen ${ }^{29}$, und dass mit F1 und G1 zwei dieser Felder gerade dem Thron gegenüber in dem Bereich liegen, den ein Besucher im Megaron nach einer Viertelumkreisung des Herdes erreicht hätte; leider können aber nicht alle Felder mit Ritzungen, zu denen die Grabungspublikation zudem nur wenige Detailinformationen bietet, in vergleichbarer Weise schlüssig erklärt werden.

So ist nach unserem derzeitigen Wissensstand die allgemeinere Erkenntnis wesentlicher, dass die Bedeutung der Querachse des Thronraumes als Blickachse eindrücklich durch die Orientierung der figürlichen Elemente des Bodenmalereien bestätigt wird, in Pylos des einzelnen Oktopus vor dem Thron ${ }^{30}$, in Tiryns der Oktopoden und Delphinpaare, die zumindest im äußeren, in Wandnähe gelegenen Teil des Megaronbodens, wahrscheinlich aber im gesamten Thronraum jedes zweites Quadratfeld zieren (Abb. 5) ${ }^{31}$. Während für erstere der Vergleich mit Vasenbildern klar auf eine korrekte Orientierung

\footnotetext{
28 Vgl. Thaler 2006 für die Unterscheidung und analytische Bedeutung dieser Kategorien.

29 Blegen - Rawson 1966, 85.

30 Blegen - Rawson 1966, 84.

31 Hackl 1912, 223 Taf. 19. Hirsch $(1980,456)$ verweist darauf, dass in Abweichung von der konservativen Rekonstruktion durch Hackl eine durchgehende Einteilung des Megaronbodens und insbesondere von dessen um den Herd gelegenen mittleren Bereich auch für Tiryns plausibel ist; bereits Rodenwaldt hatte nach Ansicht des Megaronbodens von Mykene vermerkt, dass auch in Tiryns »bei der Rekonstruktion des Planes die Quadrierung überall bis unmittelbar an den Herdrand fortgesetzt [hätte] werden müssen. Bei dem Entwurf dieses Planes war darauf verzichtet worden, weil man nicht wußte, welche künstlerische Lösung das Zusammenstoßen des Rechteckmusters mit dem Kreis gefunden habe. Jetzt zeigt es sich, daß es zu griechisch empfunden war, eine solche Lösung überhaupt zu erwarten« (Rodenwaldt 1919, 89-90). Abbildung 5 ist gegenüber der Darstellung bei Hackl entsprechend ergänzt.
} 
aus Sicht des Thronenden verweist, ließe sich im Falle der Tirynther Delphine unter Umständen die Möglichkeit in Betracht ziehen, dass diese - eher unseren Sehgewohnheiten entsprechend und wie die Oktopoden mit dem Kopf nach oben orientiert - eine gerade entgegengesetzte Orientierung aufweisen ${ }^{32}$. Ebenso ließe sich erwägen oder zumindest spekulieren, dass die eigentümliche Rasterabweichung im pylischen Megaronboden südöstlich des Herdes der Blicklenkung gedient haben könnte, in diesem Falle der Lenkung der bereits angesprochenen flüchtigen Seitenblicke vor Erreichen der Position dem Thron gegenüber33; es dürfte allerdings kaum möglich sein, ein zwingendes Argument ins Feld zu führen, diese Annahme anderen Erklärungen, z. B. der Vermutung von >Pfusch am Bau<, vorzuziehen ${ }^{34}$. So ist es unabhängig von den beiden letztgenannten Überlegungen vor allem bedeutsam, die allgemeine, in zwei Richtungen wirksame Blickachse und weniger eine konkrete Blickrichtung hervorzuheben. Diese Achse liegt quer zur Laufrichtung des in das Megaron Eintretenden ${ }^{35}$.

Und eben in der durch diese Achse vorgegebenen Blickrichtung kommt auch die auf den Sitz des Herrschers bzw. den sitzenden Herrscher bezogene Freskenkomposition am besten zur Geltung, die für die Thronwand des Megarons in Pylos belegt ist. Neben den Resten der Bankettszene mit Leierspieler, die nicht unmittelbar auf den Thronplatz bezogen ist, bildet bekanntlich ein Greif-Löwe-Paar das zweite Hauptelement der Verzierung dieser Wand, das durch aus Sicht des Betrachters links des Thrones in Versturzlage gefundene Fragmente belegt ist. Die bereits von den Ausgräbern vorgeschlagene antithetische Ergänzung dieses heraldischen Motivs durch ein weiteres Greif-Löwe-Paar rechts

32 Hackl geht indes explizit von einer einheitlichen Orientierung von Oktopoden und »nach unten tauchenden Delphinen« aus (Hackl 1912, 224. Vgl. 226. 230: »ins Wasser tauchende Delphine«) und auch Hirsch (1977, 38) teilt diese Auffassung, für die auch die Orientierung des Schuppenmusters in den nicht figürlich verzierten Feldern spricht (vgl. allerdings hierzu Hackls [1912, 228] Verweis auf Rodenwaldt [1912, 80 Anm. 1] und das von Hirsch [1980, 457] angeführte Vergleichsbeispiel in Pylos [Lang 1969, Taf. 98 d]). Eine nicht einheitliche Orientierung von Feldern findet sich bei nicht figürlicher Verzierung z. B. im Megaronhof von Mykene (Hirsch 1977, 29-30, vgl. 31 hinsichtlich unterschiedlicher Orientierung in benachbarten Raumeinheiten) und darf bei figürlicher Verzierung in Pylos für die Räume 49 und 50 vorausgesetzt werden (Blegen - Rawson 1966, 212. 214 Abb. 163).

33 Zumindest im kleinen Megaron in Tiryns könnten die von Südwesten nach Nordosten verlaufenden Diagonalen roter und gelber Felder in ähnlicher Weise den Blick des Eintretenden zum Thronplatz gelenkt haben (Hackl 1912, Taf. 20; klarer: Hirsch 1977, Taf. 11 Abb. 23). Da diese Diagonalen jedoch ein Resultat der auch an anderen Stellen regelmäßig bei einer Verschiebung um ein Feld von Reihe zu Reihe wiederholten Farbabfolge blau-rot-blau-geld (Hirsch 1977, 45) sind, ist hierin nicht zwingend eine intentionelle Blicklenkung zu sehen.

34 Hirsch $(1977,47$; Hirsch 1980, 457) verweist auf die relative Häufigkeit zumeist allerdings weniger auffälliger Unregelmäßigkeiten in Feldrastern, unter denen ein älterer Boden des Megarons in Mykene ein weiteres markantes Beispiel geben dürfte (Hirsch 1977, 31); gleichzeitig erteilt sie der These, die Abweichung resultiere aus der Schwierigkeit, im Bereich der Säulen eine Richtschnur zu spannen (Blegen - Rawson 1966, 83) eine berechtigte Absage (Hirsch 1977, 34).

35 In Abweichung hiervon ist das Schuppenmuster im kleinen Megaron auf den Eintretenden ausgerichtet (Anmerkung von Rodenwaldt in: Hackl. 1912, 222 Anm. 1). 

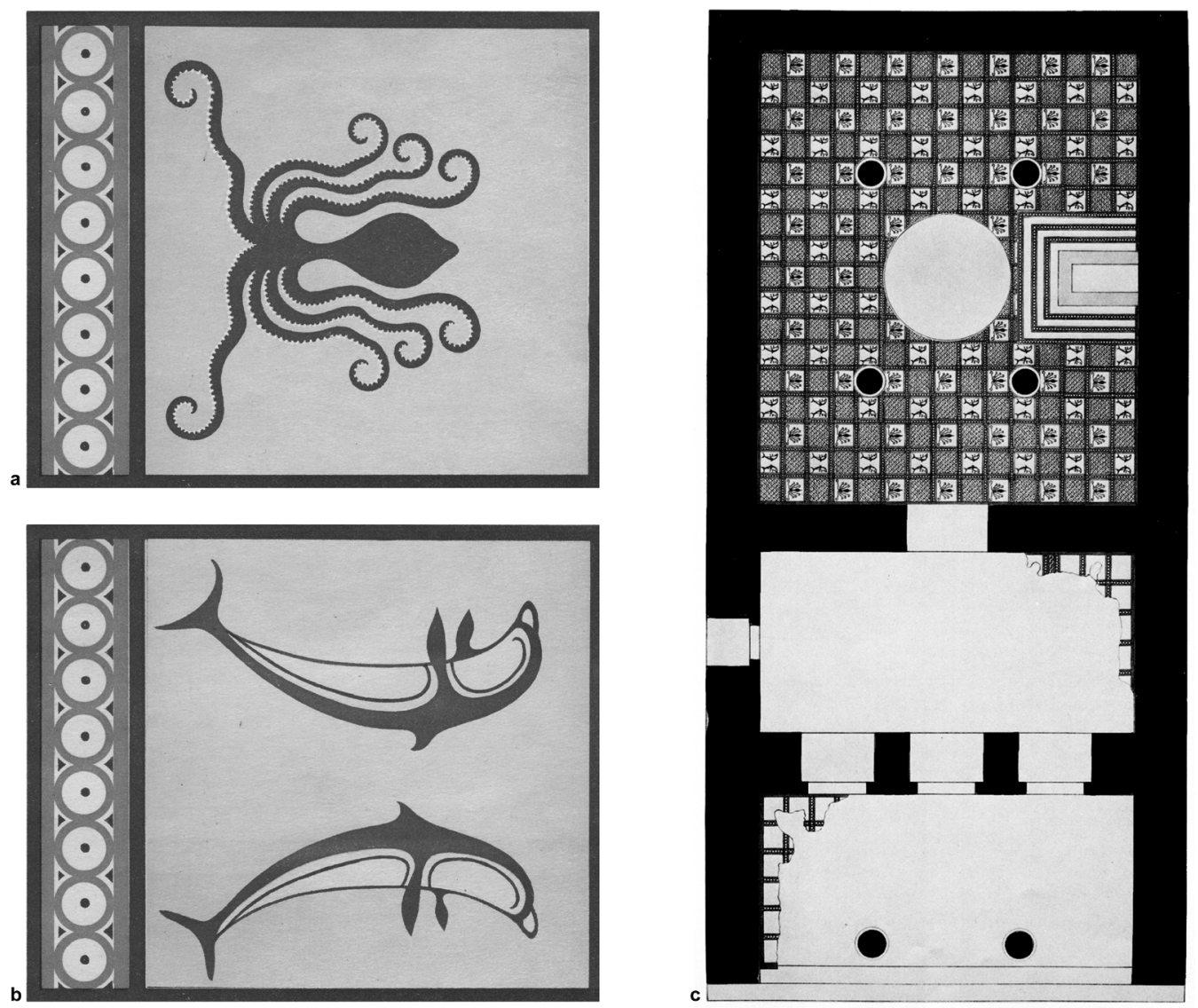

Abb. 5 |Thronraum 7 des großen Megarons im Palast von Tiryns, a: Rekonstruktion eines Fußbodenfeldes mit Darstellung eines Oktopus; b: Rekonstruktion eines Fußbodenfeldes mit Darstellung eines Delphinpaares; c: Rekonstruktion der Fußbodenmalereien.

des Thrones wurde zumeist akzeptiert ${ }^{36}$, verschiedentlich aber auch kritisch hinterfragt ${ }^{37}$. Diese Diskussion soll an dieser Stelle nicht vertieft werden und wird in Ermangelung von dem entsprechenden Wandabschnitt zuweisbaren Fragmenten von Löwen-, Greifen- oder sonstigen Darstellungen kaum abschließend zu behandeln sein; wenn ich im Folgenden eine antithetische Komposition annehme, dann nur in dem Verständnis, dass die vorgetragene Argumentation von dieser nicht abhängt. Wesentlicher ist für meine Überlegungen der enge Bezug von Malerei und Thronendem, den John Bennet besonders anschaulich herausgestellt hat:

36 Blegen 1956, 95 Taf. 40 Abb. 2; Blegen - Rawson 1966, 79; Immerwahr 1990, 136; Rehak 1995, 109.

37 Reusch 1958, 339; McCallum 1987, 97-101. 
The fact that the wanax is apparently not actually depicted on the megaron wall is interesting and suggests that the composition only >worked < when he was physically present on the seat, his authority enhanced by the >focalising < griffins and felines to either side of the seat. The composition was only complete when a live< performance was enacted. ${ }^{38}$

Der Standpunkt, von dem diese Komposition und Interaktion sich dem Betrachter vollständig erschließen, ist offenkundig die gegenüberliegende Raumseite (Abb. 6). Wie bereits ausgeführt bilden für den vom Eingang aus im Uhrzeigersinn dort angelangten Besucher die Säulen nicht länger Sichthindernisse, sondern sogar eine Rahmung des Blickes auf den Thron; genau dies gilt aber nicht nur für den Thron selbst, sondern in gleicher Weise auch für die auf ihn bezogene Freskenkomposition: Von der Mitte der Südwestwand ist zwischen der nördlichen und westlichen Säule ein beiderseits der Blickachse ca. 3,3 m breiter Abschnitt der Thronwand zu sehen, der sich bei einem Standpunkt auf halber Strecke zwischen Südwestwand und Herd auf ca. 3,7 m erweitert ${ }^{39}$. Die Greif-Löwe-Paare fallen demgegenüber in einen Bereich, der je nach Rekonstruktion mit ca. 2,6 m bis 3,2 $\mathrm{m}$ rechts und links der Thronmitte anzusetzen ist ${ }^{40}$. In die Bildkomposition wird also neben Wandmalerei und dem menschlichen Handelnden auch ein architektonischer Rahmen integriert, den in diesem Falle die Säule und auch der zwischen ihnen liegende, ebenfalls malerisch verzierte große Rundherd bilden. Ein Hitzeflimmern über letzterem mag der Figur des wanax noch eine zusätzliche Wirkung verliehen haben.

Ein letzter malerischer Befund, der in dieser Diskussion Erwähnung verdient, stammt wiederum aus Tiryns: Es sind dies die von Rudolf Hackl beim Thronpodest festgestellten und in plausibler Anlehnung an die Fußbodenmalerei aus dem kleinen Megaron um das Podest umlaufend ergänzten Zierbänder ${ }^{41}$, durch die der sichtbar aus seiner Umgebung herausgehobene Thronplatz erheblich vergrößert wird. Insbesondere - und gerade diesbezüglich ist der Parallelbefund aus dem Hauptraum des kleinen Megarons eindeutig - wird durch diese Vergrößerung die Lücke zwischen Thronplatz und Herd fast vollständig geschlossen, so dass nicht nur die Verbindung beider architektonisch betont wird, sondern auch zwischen ihnen für den hypothetischen Besucher, der McCallums

38 Bennet 2001, 34. Auch für Bennets Erläuterung gilt, dass sie im Kern, hinsichtlich des engen Bezugs von Malerei und menschlichem Akteur, nicht von der Annahme einer antithetischen Komposition abhängt gleichwohl wirkt sie im Kontext dieser Annahme, wie sein Verweis auf die »focalising < griffins and felines« verdeutlicht, besonders überzeugend.

39 Die Werte wurden auf dem bei Nelson (2001, 275 Abb. 15) abgebildeten Steinplan gemessen, wobei der dort falsch angegebene Maßstab nach dem Gesamtsteinplan des Hauptgebäudes (Nelson 2001, 274 Abb. 14) korrigiert wurde.

40 Der kleinere Wert orientiert sich an McCallums (1987, 198 Taf. 9), der größere an Youngers (1995, Taf. 76) Rekonstruktion, wobei bei letzterer der Maßstab um den Faktor 10 berichtigt und die beiden äußeren Tiere der hier als Löwe-Greif-Löwe-Dreiergruppen rekonstruierten Tierdarstellung nicht berücksichtigt wurden; selbst unter deren Einbeziehung ergibt sich nur ein Raumbedarf von ca. 3,7 m beidseitig der Mittelachse. In de Jongs Zeichnung bei Lang (1969, Taf. 125) ist leider weder der Thronplatz noch ein Maßstab angegeben.

41 Hackl 1912, 223-224 Taf. 19. 20. 

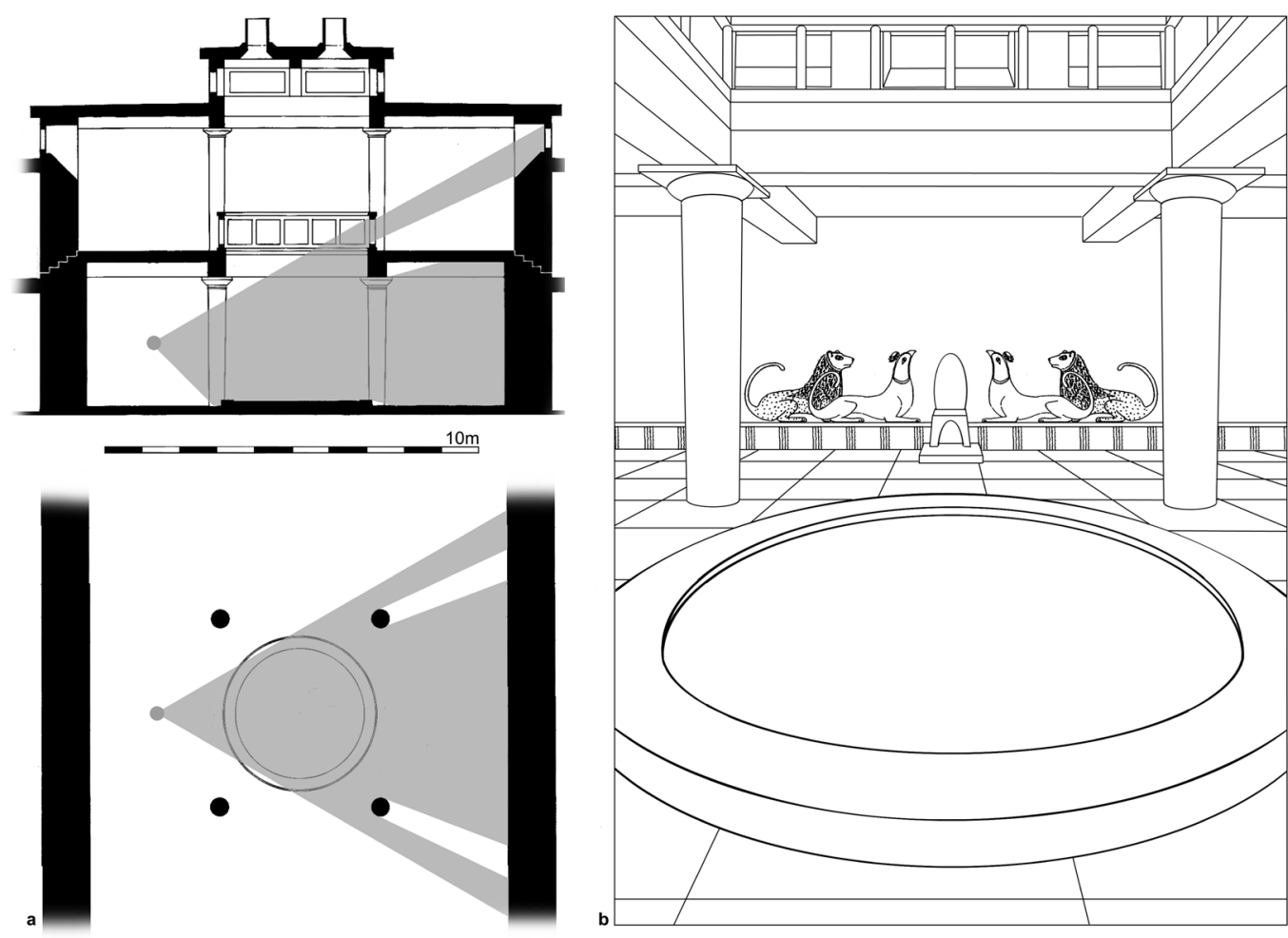

Abb. 6 | a: Querschnitt und Grundriss des mittleren Bereiches des Thronraums 6 des Palastmegarons von Pylos mit Angabe des maximalen binokularen Blickfeldes (horizontal $\pm 30^{\circ}$, vertikal $+30^{\circ} /-45^{\circ}$ ) eines dem Thron gegenüber auf halber Strecke zwischen Rundherd und Südwestwand positionierten Betrachters, Augenhöhe ca. 1,65 m (M. 1: 250); b: Rekonstruktion der Ansicht des Thronplatzes im Palastmegaron von Pylos anhand des in »a« angegebenen Blickfeldes.

Annahme folgend von der Tür direkt zum Thron schreitet, überhaupt kein Platz verbleibt, vor dem Thron Aufstellung zu nehmen. Denn obwohl eine rein ornamentale Funktion der Zierbänder in Ermangelung direkter Zeugnisse nicht vollständig auszuschließen ist, erscheint doch die Annahme unausweichlich, dass mit dieser deutlichen Markierung ein Bereich angegeben wurde, zu dem als engstem persönlichen Raum des thronenden Herrschers auch Besucher des Megarons kaum unterschiedslos, wenn überhaupt, Zugang erhielten. Im kleinen Megaron ist die westliche Begrenzung des Thronplatzes sogar nach Norden, anscheinend auch nach Süden und damit mutmaßlich über die ganze Länge des Raumes fortgesetzt, wodurch der gesamte Ostteil des Raumes mit dem Thronplatz gegenüber dem größeren Westteil abgesetzt wird ${ }^{42}$. Dass diese Unterteilung, für die im großen Megaron keine Hinweise vorliegen, bedeutsam und nicht nur Mittel etwa zu dem Zweck 
ist, eine Unregelmäßigkeit im Feldraster auszugleichen, deutet der Umstand an, dass sie ihrerseits offenbar Ursache einer Unregelmäßigkeit ist, nämlich der geringeren Breite der Felder im östlichen Raumteil ${ }^{43}$. Auch diese Beobachtungen verweisen wieder in großer Nachdrücklichkeit auf die dem Thron gegenüberliegende Seite des Saales als den Punkt, von dem aus ins Megaron vorgelassene Besucher mit dem wanax in Interaktion traten.

\section{Ideologische Komponenten eines >Gesamtkunstwerkes}

Dies führt uns zurück zum Blick von dort auf den Thron, wobei über architektonische Rahmung und Wandmalereischmuck hinaus noch die ideologischen Konnotationen des dargebotenen Bildes anzusprechen sind. Verschiedentlich ist die Säule als symbolisch aufgeladenes Element der mykenischen Architektur diskutiert worden. Beispielsweise wurde die Lesung der Säule im Löwentorrelief als Chiffre für den Palast als Ganzen vorgeschlagen 44 - freilich ein Deutungsvorschlag unter mehreren ${ }^{45}$, der an dieser Stelle indes dadurch an Attraktivität gewinnt, dass das Relief mit seinen antithetisch die Säule flankierenden mutmaßlichen Löwen eine höchst seltene monumental ausgeführte Parallele zum vermuteten antithetischen Kompositionsschema der pylischen Thronfresken bildet. Obgleich dies angesichts des Abrutschens der Thronwand im mykenischen Megaron rein spekulativ bleiben muss, ist es interessant, auf die Möglichkeit eines bildlichen Bezugs zwischen Stationen auf dem Weg zum Thron und der direkten Umgebung des Thrones zu verweisen, insbesondere, wenn die Position des Herrschers im Bildschema durch eine auf ein charakteristisches Bauelement des Thronsaales verweisende Architekturchiffre besetzt werden kann ${ }^{46}$.

Noch bedeutsamer und in ihrer Interpretation wohl weniger strittig ist die visuelle Einbeziehung des großen Rundherdes im zentralen Vordergrund des Blickfeldes. James C. Wright hat zu Recht darauf verwiesen, dass angesichts der zentralen Position und unverkennbaren Monumentalisierung des Rundherdes im Thronsaal es angemessen

43 Hirsch 1977, 37. Nach Hackls (1912, Taf. 20) Rekonstruktionszeichnung schließt diese Trennlinie näherungsweise an der rechten Türwange mit der Türangel ab, während die von ihr zusammen mit den vier östlichen senkrechten Felderreihen eingenommene Breite nahezu derjenigen von vier der westlichen Reihen entspricht.

44 Erstmals von: Adler 1865, Sp. 9-10; in der Folge ähnliche Argumentation u. a. bei: Mylonas 1957, 28; Åström - Blomé 1965, 188-189; Wright 1994, 58. Eine gute Zusammenfassung der Forschungsdiskussion bieten Åström - Blomé (1965, 160-176. 187).

45 Für die Säule als anikonisches Kultbild Stellungnahmen u. a. bei: Evans 1901, 156-158; Wace et al. 19211923,16 .

46 Querbezüge zwischen verschiedenen Stationen auf dem Weg zum Thronraum lassen sich am besten in Tiryns im Gebrauch von Konglomerat (Küpper 1996, 273 Abb. 220, 2; Maran 2006, 90 Taf. 12) und in der Fassadengestaltung der Propyla und des großen Megarons (Dörpfeld 1886, 241; Siedentopf in: Jantzen 1975, 29; Küpper 1996, 111-112) verfolgen. 
erscheint, nicht einfach, wie Klaus Kilian vorgeschlagen hatte, von einer mykenischen wanax-Ideologie zu sprechen ${ }^{47}$, sondern von einer Herd-wanax-Ideologie ${ }^{48}$. Unabhängig davon, wie man sich diesen Begriff konkret mit Leben gefüllt vorstellt - eine Anlehnung an den späteren oikos-Begriff liegt nahe, setzt aber eine Diskussion der Herdstellen in nichtpalatialen Kontexten voraus ${ }^{49}$-, ist die unmittelbare Erfahrbarkeit der engen Beziehung zwischen dem thronenden Herrscher und dem zentralen >Staatsherd im Blick durch das Megaron offenkundig von Bedeutung für die Wirkung des Konzeptes im zeitgenössischen Kontext. So erscheint wiederum zum einen der von McCallum vermutete Gang von der Thronsaaltür nach rechts, der zwischen Herd und Thron führt, in hohem Maße unplausibel und zum anderen das von Bennet angedeutete Bild dahingehend ergänzt, dass nicht nur Wandmalerei, menschliche(r) Handelnde(r) und, wie zuvor angemerkt, architektonische Rahmung, sondern auch die symbolischen Konnotationen der architektonischen Elemente in gleichem Maße wie diejenigen der Wandbilder zu einem >Gesamtkunstwerk< arrangiert waren.

\section{Der Kreis schließt sich (für wenige)}

Mit den bisherigen Ausführungen dürften die Annahme einer Bewegung von der Thronraumtür nach links und die Bedeutung der dem Thron gegenüberliegenden Raumseite für Besucher hinlänglich begründet sein; zu behandeln bleibt die Fortsetzung des Weges zum Thron im Sinne der zuvor postulierten Bewegung im Uhrzeigersinn um den Zentralherd. Hierzu ist zunächst anzumerken, dass keinesfalls davon auszugehen ist, dass jedem in den Thronsaal vorgelassenen Besucher ein weiteres Vordringen in Richtung des Thrones und Herrschers gestattet war; es sei in diesem Kontext beispielhaft an die Verlängerung der malerischen Thronplatzbegrenzung über die gesamte Ostseite des kleinen Tirynther Megarons erinnert. Die allgemeine Bedeutung abgestufter Zugangsrechte für das Verständnis der mykenischen Palastarchitektur ist in der jüngeren Vergangenheit mit zunehmender Klarheit herausgearbeitet worden: Wright hat die Architektur der Paläste als »a series of concentric rings of symbols that increasingly focus attention on the [...] megaron with its monumental hearth and royal throne « ${ }^{50}$ beschrieben und im selben Zusammenhang z. B. für den Tirynther Palasthof Zugangsbeschränkungen vermutet ${ }^{51}$; Lisa Bendall hat anhand der Trinkgefäße aus Pylos sehr anschaulich dargestellt, wie eine Abstufung von Zugangsrechten bei festlichen Anlässen zum Tragen kam und selbst über bewegliche Funde verfolgt werden kann, obgleich ihre Studie hinsichtlich der

47 Kilian 1988.

48 Wright 1994, 56-59.

49 Tournavitou 1999.

50 Wright 1994, 51.

51 Wright 1994, 60. 
Materialgrundlage nicht unbestritten geblieben ist ${ }^{52}$; ich selbst habe unter Bezug auf transägäische architektonische Parallelen und unter Heranziehung hethitischer Textquellen darauf verwiesen, wie Abstufungen von Zugangsrechten sich in konkreten Handlungen ausdrücken konnten und nicht nur festliche Anlässe, sondern auch das alltägliche Handeln prägten ${ }^{3}$. Vor diesem Hintergrund erscheint die Annahme nur folgerichtig, dass auch der Bereich gegenüber dem Thron für einen Teil und vielleicht den größeren Teil derer, die Eintritt in den Thronsaal erlangten, den Schluss- bzw. Umkehrpunkt ihres Weges darstellte. Dass damit schon aus raumtheoretischer Sicht, aufgrund der positiven Korrelation zwischen der Öffentlichkeit des Kontextes und der Redundanz architektonischer Hinweisgeber ${ }^{54}$, eine geringere archäologische Sichtbarkeit der fortgeführten Bewegung zum Thron hin zu erwarten steht und sich die Befundlage auch in der Praxis als weniger eindeutig erweist als die zuvor hinsichtlich der Orientierung von der Thronsaaltür nach links dargestellte Situation, soll jedoch nicht davon abhalten, eine Diskussion von Aspekten der weiteren Weggestaltung zu versuchen.

In Pylos sind zwei mögliche Wegestationen bei der fortgesetzten Annäherung an den Thron zu fassen: der bei der westlichen Säule gefundene dreifüßige Opfertisch und die direkt nordwestlich des Thronplatzes befindliche sog. Libationseinrichtung55. Obschon es leicht fiele, konkrete Handlungsabläufe unter Einbeziehung beider Elemente vorzuschlagen, muss einschränkend aber direkt angemerkt werden, dass beim Opfertisch als nicht-fixem, also mobilem Element Auffindungs- und Nutzungsort(e) nicht identisch sein müssen, wobei die Auffindung von Miniaturkylikes in situ auf dem Opfertisch deutlich hierfür spricht; selbst bei einer Nutzung in der Fundposition bleibt aber eine Einordnung in den Kontext der Huldigung des wanax von der dem Thron gegenüberliegenden Seite des Herdes denkbar. Bei der Libationseinrichtung, die aus zwei vor der Nordostwand gelegenen, durch ein schmale Rinne verbundenen runden Vertiefungen im Stuckboden besteht, handelt es sich hingegen um ein fixes Element, das bislang hauptsächlich mit dem Herrscher und durch ihn ausgebrachte Trankopfer in Beziehung gesetzt wurde. Die symmetrische Gestalt der Installation verdeutlicht jedoch, dass ein Ausgießen eines Trankopfers, womöglich sogar für den wanax selbst ${ }^{5}$, durch einen seitlich an den Thron tretenden Besucher oder auch ein gemeinsames Darbringen einer Libation durch Thronenden und Besucher ebenso in Erwägung gezogen werden kann.

Nicht unproblematisch ist aufgrund der insbesondere durch den Tirynther Megaronboden verdeutlichten starken Hervorhebung des erweiterten Thronplatzes aus seiner Umgebung die Frage nach dem letzten Standpunkt der in die Nähe des Herrschers vorgelassenen Besucher. Durften diese nicht direkt vor den Thron - und damit in den Bereich

52 Bendall 2004, 112-124; Kritik insbesondere bei Hruby 2006, 109 Anm. 40.

53 Thaler 2007, 304-305.

54 Hall 1966, 96; Rapoport 1982, 84. 117. 145. 149-152; Sanders 1984, 93. 439-440. 545.

55 Blegen - Rawson 1966, 88.

56 Vgl. Säflund 1980, 241. 
der malerischen Thronplatzmarkierung - treten und mussten folglich nach einer letzten Station seitlich des Thrones aus einer Sackgasse umkehren und den zum Thron hin genommenen Weg zurückverfolgen? Bestand hier eine weitere Abstufung von Zugangsrechten? Antworten bleiben hier wohl spekulativ. Am wahrscheinlichsten ist meines Erachtens, dass nur ein kleiner und sehr exklusiver Kreis von Besuchern überhaupt auf die Thronwandseite der Megaronhaupträume vorgelassen wurde und dass diese dann auch in die unmittelbare Umgebung des wanax eintreten durfte - ob diese nun, wie in Tiryns, malerisch klar herausgehoben oder, wie mutmaßlich in Pylos, nur durch die Präsenz des Herrschers selbst definiert war -, die sie danach in Richtung der Thronsaaltür wieder verließen.

\section{Ausblick: Weiterführende Überlegungen und ein Perspektivwechsel}

Unabhängig von der Beantwortung dieser letzten Fragen scheint die grundsätzliche Akzeptanz einer Bewegung im Uhrzeigersinn um den Zentralherd herum oder zumindest einer entsprechenden primären Orientierung des Besucherverkehrs in den Haupträumen der mykenischen Palastmegara unausweichlich. Diese Erkenntnis ist sowohl per se von Interesse als auch hinsichtlich neuer Perspektiven, die sie eröffnet und von denen hier nur zwei kurz angedeutet werden sollen.

Zum einen stellt sich die Frage, ob die Kreisbewegung, die in einem architektonischen Kontext, der eine strenge Kontrolle sozialer Normen erwarten lässt, an dem symbolisch ebenso wie räumlich zentralen Rundherd orientiert ist, über ihre Rolle in der wahrnehmungsmäßigen Erschließung dieses baulichen Kontextes für den Besucher hinaus nicht selbst symbolisch aufgeladen ist und z. B. eine kosmologische Bedeutung transportiert. Obschon in einem nichtstaatlichen Kontext verankert, liefert etwa das seinerseits u. a. durch ethnographische Schilderungen inspirierte sun-wise model, das zur Erklärung der Struktur eisenzeitlicher britischer Rundhäuser vorgeschlagen wurde ${ }^{57}$, ein gutes illustratives Beispiel der Art von Phänomenen, an die sich hier denken lässt. Konkrete Vorschläge dürften jedoch in Anbetracht der für die mykenische Kultur verfügbaren Quellen schwer zu untermauern sein.

Zum anderen liegt die Vermutung nahe, dass mit der Bewegungsrichtung im Thronsaal auch die Leserichtung der dort angebrachten Wandmalereien zusammenfällt, in ähnlicher Weise wie dies im assyrischen Kontext Barbara N. Porter für die Thronsaalreliefs im Nordwestpalast von Kalhu dargestellt hat $t^{8}$. Angesichts der Anlehnung an die Wahrnehmung des sich in der Architektur bewegenden Besuchers dürfte diese Annahme letztlich plausibler sein als die von mir an anderer Stelle für das Megaron von Pylos angedachte, eher - wenn auch nicht ausschließlich - aus der Vogelperspektive der Grund-

57 Fitzpatrick 1994.

58 Porter 2003, 182-188. 
rissbetrachtung schlüssige Möglichkeit einer Lesung im Sinne einer binären Opposition zweier in innerer und äußerer Hälfte des Thronsaales repräsentierter Konzepte ${ }^{59}$. Eine Überlagerung und Komplementarität dieser beiden und unter Umständen noch anderer Leseweisen ist natürlich denkbar. Gemeinsam ist beiden Lesevorschlägen in jedem Falle leider die Schwierigkeit, sie trotz der lückenhaften oder gänzlich fehlenden Überlieferung der Wandmalereien in den Palastmegara in Pylos und Tiryns als Grundlage einer konkreten, detaillierten Lesung heranzuziehen. Umso erfreulicher ist die Übereinstimmung, die sich mit der von Gerhart Rodenwaldt bereits vor langer Zeit vorgestellten Interpretation der Thronraumfresken von Mykene ergibt. Rodenwaldt erschließt nach Motivik und Fundlage der erhaltenen Fragmente eine Darstellung, die von der Tür aus über den nördlichen Teil der Westwand und die Nordwand hinweg »von links nach rechts abzulesen ist $^{6}{ }^{60}$ und in einer »fortschreitende[n] Handlung [...] die Rüstung der Wagen im Lager, die Fahrt zum Kampf und die Kampfszenen selbst « $^{61}$ als aufeinander folgende Episoden einer Schlacht schildert. Obwohl die Fortsetzung des Frieses durch den Absturz der Ost- und Südwand des Megarons in die Chavos-Schlucht verloren ist und Rodenwaldt letztlich nur die Lagerszene links der Tür und die Schlachtdarstellung, die Streitwagen und Fußsoldaten umfasst und wohl um eine große Architekturzeichnung in der Mitte der Osthälfte der Nordwand angeordnet war, klar belegen und verorten $\mathrm{kann}^{62}$, während für die »Fahrt zum Kampf« klare Indizien fehlen, ist die Identifikation der Leserichtung überzeugend und somit die Parallele zur hier vorgestellten Argumentation augenscheinlich ${ }^{6}$.

Während sich trotz dieser Parallele in den beiden zuletzt angesprochenen Punkten insgesamt zwar Perspektiven, aber noch keine eingehenderen Interpretationen erschließen, sei zum Schluss ein Perspektivwechsel versucht, der ein letztes Indiz zur Stützung der Hauptthese dieses Aufsatzes liefern mag und zurück an einen der Ausgangspunkte der obigen Überlegungen führt, genauer gesagt zu der in den Wandmalereien, wie Fragmentkomplex 45 H 6, reflektierten Bedeutung von Prozessionen innerhalb des mykenischen Hofzeremoniells. Nachdem zuvor hauptsächlich das sich dem Besucher darbietende Bild des Thronraums behandelt wurde, ist zur Perspektive des thronenden Herrschers anzumerken, dass eine Reihe von Gabenträgern, die sich hypothetisch von der Tür des Thronsaals direkt auf seinen Sitz zu bewegten, für den wanax zwar zeitlich als Folge, aber nicht visuell als Prozession erfahrbar wäre. Eine durch das >Fenster zwischen den Säulen auf der Gegenseite des Saales schreitende Gabenträgerreihe wäre hingegen als Prozession im Wortsinne sersichtlich $<$ - und böte ein Bild, das direkt der Darstellung der Wandmalereien entspräche.

59 Thaler 2006, 102.

60 Rodenwaldt 1921, 44.

61 Rodenwaldt 1921, 59 .

62 Rodenwaldt 1911, 245-247; Rodenwaldt 1921, 24-45, bes. 29. 43.44.

63 Hierfür ist $u$. a. bedeutend, dass die Lagerszene nicht nur, wie von Immerwahr $(1990,123)$ verkürzend wiedergegeben, allgemein der Westwand, sondern, wie Rodenwaldt (1921, 29. 44) an zwei Stellen betont, im Speziellen dem links der Tür gelegenen Nordabschnitt der Westwand zuzuweisen ist. 


\section{Bibliographie}

\section{Adler 1865}

F. Adler, Das Relief am Löwenthor zu Mykenae, AZ 23, 1865, Sp. 1-13.

\section{Åström - Blomé I965}

P. Åström - B. Blomé, A Reconstruction of the Lion Relief at Mycenae, OpAth 5, 1965, 159-191.

\section{Bendall 2004}

L. Bendall, Fit For a King? Hierarchy, Exclusion, Aspiration and Desire in the Social Structure of Mycenaean Banqueting, in: P. Halstead - J. C. Barrett (Hgg.), Food, Cuisine and Society in Prehistoric Greece. Papers Presented to the 6th Round Table of the Sheffield Centre for Aegean Archaeology, Sheffield 2001, Sheffield Studies in Aegean Archaeology 5 (Oxford 2004) 105-135.

\section{Bennet 200I}

J. Bennet, Agency and Bureaucracy. Thoughts on the Nature and Extent of Administration in Bronze Age Pylos, in: S. Voutsaki - J. Killen (Hgg.), Economy and Politics in the Mycenaean Palace States. Proceedings of a Conference Held on 1-3 July 1999 in the Faculty of Classics, Cambridge, Cambridge Philological Society Supplementary Volume 27 (Cambridge 2001) 25-37.

\section{Blegen 1956}

C. W. Blegen, The Palace of Nestor Excavations of 1955, AJA 60, 1956, 95-101.

\section{Blegen - Rawson I966}

C. W. Blegen - M. Rawson, The Palace of Nestor at Pylos in Western Messenia I. The Buildings and Their Contents (Princeton 1966).

\section{Brecoulaki 2005}

H. Brecoulaki, Conservation and Re-Study Project of the Mycenaean Wall Paintings, in: The Pylos Regional Archaeological Project. 15th Season Preliminary Report the the 7th Ephoreia of Prehistoric and Classical Antiquities, Olympia, on the Results of Museum Study, September 2004 - October 2005 (2005), <http://classics.uc.edu/prap/reports/ HARP2005.html\#body.s_divo.4_div1.2> (11.03.2012).

\section{Cameron 1970}

M. A. S. Cameron, New Restorations of Minoan Frescoes from Knossos (Summary), BICS 17, 1970, 163-166. 


\section{Darcque 1990}

P. Darcque, Pour l'abandon du terme >mégaron<, in: P. Darcque - R. Treuil (Hgg.), L'habitat égéen préhistorique. Actes de la table ronde internationale, Athènes, 23-25 juin 1987, BCH Suppl. 19 (Athen 1990) 21-31.

\section{Dörpfeld I886}

W. Dörpfeld, Die Bauwerke von Tiryns, in: H. Schliemann, Tiryns. Der prähistorische Palast der Könige von Tiryns. Ergebnisse der neuesten Ausgrabungen (Leipzig 1886) $200-352$.

\section{Evans I90I}

A. J. Evans, Mycenaean Tree and Pillar Cult and Its Mediterranean Relations, JHS 21, 1901, 99-204.

\section{Fitzpatrick 1994}

A. Fitzpatrick, Outside in. The Structure of an Early Iron Age House at Dunston Park, Thatcham, Berkshire, in: A. Fitzpatrick - E. Morris (Hgg.), The Iron Age in Wessex. Recent Work (Salisbury 1994) 68-72.

\section{Graham I960}

J. W. Graham, Mycenaean Architecture. Archaeology 13, 1960, 46-54.

\section{Hackl I9I2}

R. Hackl, Die Fußböden, in: G. Rodenwaldt, Die Fresken des Palastes, Tiryns. Die Ergebnisse der Ausgrabungen des Instituts 2 (Athen 1912) 222-238.

\section{Hall 1966}

E. T. Hall, The Hidden Dimension. Man's Use of Space in Public and Private (London $1966)$.

\section{Hirsch 1977}

E. S. Hirsch, Painted Decoration on the Floors of Bronze Age Structures on Crete and the Greek Mainland, SIMA 53 (Göteborg 1977).

\section{Hirsch 1980}

E. S. Hirsch, Another Look at Minoan and Mycenaean Interrelationships in Floor Decoration, AJA 84, 1980, 453-462.

\section{Hruby 2006}

J. A. Hruby, Feasting and Ceramics. A View from the Palace of Nestor at Pylos

(Diss. University of Cincinnati 2006).

\section{Immerwahr I990}

S. A. Immerwahr, Aegean Painting in the Bronze Age (University Park 1990). 


\section{Jantzen 1975}

U. Jantzen (Hg.), Führer durch Tiryns (Athen 1975).

\section{Johnson 2002}

M. H. Johnson, Behind the Castle Gate. From Medieval to Renaissance (London 2002).

\section{Jung 2000}

R. Jung, Das Megaron - ein Analogie(kurz)schluss der ägäischen Archäologie, in: A. Gramsch (Hg.), Vergleichen als archäologische Methode. Analogien in den Archäologien. Mit Beiträgen einer Tagung der Arbeitsgemeinschaft Theorie (T-AG) und einer kommentierten Bibliographie, BARIntSer 825 (Oxford 2000) 71-95.

\section{Kilian 1988}

K. Kilian, The Emergence of wanax Ideology in the Mycenaean Palaces, OxfJA 7/3, 1988, 291-302.

\section{Kottak 1978}

C. P. Kottak, Rituals at McDonald's. Natural History 87, 1978, 74-83.

\section{Küpper 1996}

M. Küpper, Mykenische Architektur. Material, Bearbeitungstechnik, Konstruktion und Erscheinungsbild, Internationale Archäologie 25 (Espelkamp 1996).

\section{Lang 1969}

M. L. Lang, The Palace of Nestor at Pylos in Western Messenia II. The Frescoes (Princeton 1969).

\section{Maran 2004}

J. Maran, Architektonische Innovation im spätmykenischen Tiryns. Lokale Bauprogramme und fremde Kultureinflüsse, in: Althellenische Technologie und Technik. Von der prähistorischen bis zur hellenistischen Zeit mit Schwerpunkt auf der prähistorischen Epoche. Tagung 21.-23.03.2003 in Ohlstadt/Obb. Deutschland (Weilheim 2004) 261-293.

\section{Maran 2006}

J. Maran, Mycenaean Citadels as Performative Space, in: J. Maran - C. Juwig H. Schwengel - U. Thaler (Hgg.), Constructing Power - Architecture, Ideology and Social Practice. Internationales Symposion Heidelberg 18.-20.6.2005, Geschichte. Forschung und Wissenschaft 19 (Münster 2006) 75-91.

\section{Maran im Druck}

J. Maran, Architektonischer Raum und soziale Kommunikation auf der Oberburg von Tiryns - Der Wandel von der mykenischen Palastzeit zur Nachpalastzeit, in: F. Arnold A. Busch - R. Haensch - U. Wulf-Rheidt (Hgg.), Orte der Herrschaft, Menschen Kulturen - Traditionen. Studien aus den Forschungs-Clustern des DAI 3 (Berlin im Druck). 


\section{McCallum 1987}

L. R. McCallum, Decorative Program in the Mycenaean Palace of Pylos. The Megaron Frescoes (Diss. University of Pennsylvania 1987).

\section{Müller I930}

K. Müller, Die Architektur der Burg und des Palastes. Tiryns. Die Ergebnisse der Ausgrabungen des Instituts 3 (Augsburg 1930).

\section{Mylonas 1957}

G. E. Mylonas, Ancient Mycenae. The Capital City of Agamemnon (Princeton 1957).

\section{Nelson 200I}

M. C. Nelson, The Architecture of Epano Englianos, Greece (Diss. University of Toronto 2001).

\section{Porter 2003}

B. N. Porter, Intimidation and Friendly Persuasion. Re-Evaluating the Propaganda of Ashurnasirpal II, Eretz-Israel 27, 2003, 180-191.

\section{Rapoport 1982}

A. Rapoport, The Meaning of the Built Environment. A Nonverbal Communication Approach (Beverly Hills 1982).

\section{Rehak I995}

P. Rehak, Enthroned Figures in Aegean Art and the Function of the Mycenaean Megaron, in: P. Rehak (Hg.), The Role of the Ruler in the Prehistoric Aegean. Proceedings of a Panel Discussion Presented at the Annual Meeting of the Archaeological Institute of America, New Orleans, Louisiana, 28 December 1992, Aegaeum 11 (Liège 1995) 95-118.

\section{Reusch 1956}

H. Reusch, Die zeichnerische Rekonstruktion des Frauenfrieses im böotischen Theben, AbhBerlin 1955 Nr. 1 (Berlin 1956).

\section{Reusch $195^{8}$}

H. Reusch, Zum Wandschmuck des Thronsalles in Knossos, in: E. Grumach (Hg.), Minoica. Festschrift zum 8o. Geburtstag von Johannes Sundwall, Deutsche Akademie der Wissenschaften zu Berlin. Schriften der Sektion für Altertumswissenschaften 12 (Berlin 1958) 334-356. 


\section{Rodenwaldt I9II}

G. Rodenwaldt, Fragmente mykenischer Wandgemälde, AM 36, 1911, 221-250.

\section{Rodenwaldt I9ı2}

G. Rodenwaldt, Die Fresken des Palastes, Tiryns. Die Ergebnisse der Ausgrabungen des Instituts 2 (Athen 1912).

\section{Rodenwaldt I9I9}

G. Rodenwaldt, Mykenische Studien. JdI 34, 1919, 87-106.

\section{Rodenwaldt I92I}

G. Rodenwaldt, Der Fries des Megarons von Mykenai (Halle 1921).

\section{Säflund 1980}

G. Säflund, Sacrificial Banquets in the >Palace of Nestor<, OpAth 13, 1980, 237-246.

\section{Sanders 1984}

D. H. Sanders, Behavior and the Built Environment. An Interpretive Model for the Analysis of Architecture in an Archaeological Context and Its Testing on Material from the Aegean Bronze Age Site of Myrtos, Crete (Diss. Columbia 1984).

\section{Stocker - Davis 2004}

S. R. Stocker - J. D. Davis, Animal Sacrifice, Archives, and Festing at the Palace of Nestor, in: J. C. Wright (Hg.), The Mycenaean Feast, Hesperia 73/2 (Princeton 2004) 59-75.

\section{Thaler 2006}

U. Thaler, Constructing and Reconstructing Power. The Palace of Pylos, in: J. Maran -

C. Juwig - H. Schwengel - U. Thaler (Hgg.), Constructing Power - Architecture, Ideology and Social Practice. Internationales Symposion Heidelberg 18.-20.6.2005, Geschichte. Forschung und Wissenschaft 19 (Münster 2006) 93-116.

\section{Thaler 2007}

U. Thaler, Aḩhijawa and Hatti. Palatial Perspectives, in: S. Antionadou - A. Pace (Hgg.), Mediterranean Crossroads. Internationale Konferenz Athen 10.-13.5.2005 (Athen 2007) 291-323.

\section{Tournavitou I999}

I. Tournavitou, Hearths in Non-Palatial Settlement Contexts. The LBA Period in the Peloponnese, in: P. P. Betancourt - V. Karageorghis - R. Laffineur - W.-D. Niemeier (Hgg.), Meletemata. Studies in Aegean Archaeology Presented to Malcolm H. Wiener as he Enters his 65th Year III, Aegaeum 20 (Liège 1999) 833-840. 
Wace et al. I92I-I923

A. J. B. Wace - C. A. Boethius - W. A. Heurtley - L. B. Holland - W. Lamb, Excavations at Mycenae, BSA 25, 1921-1923, 1-434.

\section{Wright 1994}

J. C. Wright, James C, The Spatial Configuration of Belief. The Archaeology of

Mycenaean Religion, in: S. E. Alcock - R. Osborne (Hgg.), Placing the Gods. Sanctuaries and Sacred Space in Ancient Greece (Oxford 1994) 37-78.

\section{Younger 1995}

J. G. Younger, The Iconography of Rulership in the Aegean: A Conspectus, in:

P. Rehak (Hg.), The Role of the Ruler in the Prehistoric Aegean. Proceedings of a Panel

Discussion Presented at the Annual Meeting of the Archaeological Institute of America, New Orleans, Louisiana, 28 December 1992, Aegaeum 11 (Liège 1995) 151-211.

\section{Abbildungsnachweis}

Abb. 1 a: Photographie A. Brysbaert, digitale Rekonstruktion der Frontalansicht U. Thaler; Abb. 1 b: nach Rodenwaldt 1912, Taf. 8; Abb. 2: nach Graham 1960, 52 Abb. 12; Abb. 3: nach McCallum 1987, 195-197 Taf. 8 a-c, digitale Zusammensetzung M. Kostoula; Abb. 4: Zeichnung H. Sulze, reproduziert mit Genehmigung des Tiryns-Projektes des DAI; Abb. 5 a: nach Rodenwaldt 1912, Taf. 21, 4; Abb. 5 b: nach Rodenwaldt 1912, Taf. 21, 3; Abb. 5 c: nach Rodenwaldt 1912, Taf. 19; Abb. 6 a: nach Blegen - Rawson 1966, Taf. 418; Abb. 6 b: Zeichnung U. Thaler. 